\title{
Multicollinearity is a red herring in the search for moderator variables: A guide to interpreting moderated multiple regression models and a critique of Iacobucci, Schneider, Popovich, and Bakamitsos (2016)
}

\author{
Gary H. McClelland ${ }^{1}$ • Julie R. Irwin ${ }^{2}$ • David Disatnik ${ }^{3} \cdot$ Liron Sivan $^{4}$
}

Published online: 16 August 2016

(C) Psychonomic Society, Inc. 2016

\begin{abstract}
Multicollinearity is irrelevant to the search for moderator variables, contrary to the implications of Iacobucci, Schneider, Popovich, and Bakamitsos (Behavior Research Methods, 2016, this issue). Multicollinearity is like the red herring in a mystery novel that distracts the statistical detective from the pursuit of a true moderator relationship. We show multicollinearity is completely irrelevant for tests of moderator variables. Furthermore, readers of Iacobucci et al. might be confused by a number of their errors. We note those errors, but more positively, we describe a variety of methods researchers might use to test and interpret their moderated multiple regression models, including two-stage testing, mean-centering, spotlighting, orthogonalizing, and floodlighting without regard to putative issues of multicollinearity. We cite a number of recent studies in the psychological literature in which the researchers used these methods appropriately to test, to interpret, and to report their moderated multiple regression models. We conclude with a set of recommendations for the analysis and reporting of moderated multiple regression that should help researchers better understand their models and facilitate generalizations across studies.
\end{abstract}

Keywords Moderated multiple regression · Interactions · Multicollinearity $\cdot$ Regression analysis $\cdot$ Tutorial

Gary H. McClelland

gary.mcclelland@colorado.edu

1 Department of Psychology and Neuroscience, CB 345, University of Colorado Boulder, Boulder, CO 80309-0345, USA

2 University of Texas, Austin, TX, USA

3 Tel Aviv University, Tel Aviv, Israel

4 Carnegie Mellon University, Pittsburgh, PA, USA
Saunders $(1955,1956)$ introduced moderated multiple regression (MMR), a simple but very general statistical method for determining whether the relationship between two variables, say $Y$ and $X$, depends on or is moderated by a third variable $Z$. The analysis determines whether adding the product $X Z$ to an additive regression model (ADD) containing the components $X$ and $Z$ significantly increases the explained variation or, equivalently, whether the coefficient for the product $X Z$ is statistically significant. Specifically, for these two estimated models

$$
\begin{aligned}
& \text { ADD }: \quad \hat{Y}=b_{0}+b_{1} X+b_{2} Z \\
& \text { MMR : } \hat{Y}=c_{0}+c_{1} X+c_{2} Z+c_{3} X Z,
\end{aligned}
$$

the test of the difference $R_{M M R}^{2}-R_{A D D}^{2}$ assesses moderation. Furthermore, $c_{3}$ estimates the raw magnitude of the moderation, testing the null hypothesis $c_{3}=0$ is an equivalent test of moderation, and its magnitude is indexed by standardized effect size measures such as the squared partial correlation $\left(\mathrm{pr}^{2}\right.$ or $\eta^{2}$ ) or the squared semi-partial correlation $\left(s r^{2}\right)$, the latter being equivalent to the difference $R_{M M R}^{2}-R_{A D D}^{2}$. The model is agnostic as to which variable is moderating the other so researchers often simply refer to a significant contribution from the product $X Z$ as an "interaction." Interactions form the basis for many psychological theories and data analyses. For example, Riglin et al. (2016), investigated whether the relationship between stress $(X)$ and depressive symptoms $(Y)$ was buffered or moderated by cognitive ability ( $\mathrm{Z}$, a continuous variable) and by gender $(\mathrm{G}$, a dichotomous variable).

Iacobucci, Schneider, Popovich, and Bakamitsos (2016, this issue, hereinafter ISPB) attempt to sort out various multicollinearity issues in the testing of moderated regression models. Unfortunately their attempt is substantially flawed and is likely to leave many readers confused about how best to test moderated regression models. The reasoning of ISPB 
appears to fall prey to the danger of inappropriately applying heuristics learned for additive regression to moderated multiple regression (Irwin \& McClelland, 2001). Metaphorically, multicollinearity is like a red herring in a mystery novel: seemingly guilty, but actually innocent, wasting the time of the detective in the search for the culprit. The arguments in ISPB ignore the difference between a stochastic relationship such as the correlation between the two predictors (e.g., stress and cognitive ability, $r_{X Z}$, or stress and gender, $r_{X G}$, in the example above) and the structural relationship between the predictors and their product (e.g., stress, $X$, as a component of the product stress * cognitive ability, $X Z$ ). As we will show below, the correlation induced by the structural ${ }^{1}$ relationship can always be reduced to zero by change-of-origin transformations whereas stochastic relationships are invariant to such transformations. Another serious error throughout their paper is references to the coefficients $c_{1}$ and $c_{2}$ from MMR as "main effects" when, as shown below, they are actually conditional effects when the other predictor is zero. Their numerical example attempting to elucidate the role of multicollinearity in a model with interactions is unhelpful because there is no interaction in their example $(b=-0.063, t(26)=-0.53, p=0.60)$. We provide a more informative numerical example below with a statistically significant interaction. Finally, ISPB's method for estimating standardized regression coefficients in MMR is incorrect.

We will throughout provide more details on the failings of ISPB as specific issues are discussed. However, our primary intent is to provide positive guidance for researchers using MMR to understand how relationships among their variables influence the accuracy and especially the interpretability of the coefficients estimated by MMR.

Some researchers are disconcertingly surprised by the sometimes dramatic change in coefficients for $X$ and $Z$ between the ADD and MMR models and have sometimes mistakenly attributed the changes to multicollinearity. For whatever reasons, this mistake has been more of an issue in the marketing and management literature than in recent psychology research. We provide numerous contemporary examples below of how psychology researchers are appropriately using MMR without regard to multicollinearity issues. Unfortunately, ISBP perpetuate the myth that multicollinearity, whether "micro" or "macro," has anything to do with the appropriate use of MMR and interpretation of its coefficients and standard errors. In the following we attempt to reduce the confusion sown by ISBP and others by examining the correct strategies to use when employing MMR, and then focus on what does and does not influence

\footnotetext{
"Our use of "stochastic" and "structural" relationship correspond roughly to ISPB's use of "essential" and "nonessential" collinearity. We avoid those terms because, as ISPB note, they are pejorative, and, more importantly, they beg the issue that the structural relationship has anything to do with multicollinearity.
}

the accuracy (i.e., standard errors) of the parameter estimates in MMR. For example, we will discuss how correlation among the predictor variables $X$ and $Z$ can enhance the statistical power for detecting the interaction.

\section{Interpreting coefficients in MMR}

We begin by examining the change in the coefficients between ADD and MMR that is the essence of an interaction: different slopes for different levels of the other variable or for different groups defined by the other variable. Rearranging and factoring the terms of the moderated model

MMR : $\hat{Y}=\left(c_{0}+c_{2} Z\right)+\left(c_{1}+c_{3} Z\right) X$

shows that the slope (i.e., the partial derivative) relating $X$ to $Y$ is $\left(c_{1}+c_{3} Z\right)$, which, as it must, depends on the level of $Z$. Whereas $b_{1}$ in the additive model estimates the average partial relationship between $X$ and $Y$ controlling for or holding constant $Z$ at any level of $Z$, the coefficient $c_{1}$ in the moderated model estimates the conditional relationship between $X$ and $Y$ when and only when $Z=0$. If zero is outside the range of the values of $Z$, then $c_{1}$ estimates a meaningless simple effect (Cohen, 1978, uses the term "arbitrary nonsense"). In the example above from Riglin et al. (2016) the coefficient for $X$ (stress) would represent the relationship between stress and depression $(Y)$ when and only when $Z$ (cognitive ability) equals zero, surely a highly unlikely value. ${ }^{2}$ ISPB confuse this issue by referring to $X$ and $Z$ as "main effect variables." Variables are not main effects; regression coefficients for those variables may either be partial relationships (i.e., the coefficients $b_{1}$ and $b_{2}$ from the ADD model, known as "main effects" in ANOVA) or only conditional relationships (known as "simple effects" in ANOVA) at a fixed level of the other variable (i.e., the coefficients $c_{1}$ and $c_{2}$ from MMR). ISPB never use the term "simple effect" even though that is primarily what they are talking about rather than the "main effects" they reference. The generalization of the concept of "main effect" from balanced experimental designs to regression models is tenuous and best avoided (Irwin \& McClelland, 2001). This is especially the case in moderated regression models where the predictor coefficients focus on conditional relationships. We prefer not to even use the term "effect" borrowed from ANOVA because it implies a causal relationship based on an experimental design with random assignment. For observational studies, we can only assess relationships,

\footnotetext{
${ }^{2}$ In the two data sets, 0 on the cognitive ability measure they used would have been either -7.3 or -8.6 standard deviations below the mean. Riglin et al. (2016) do not report the minimum scores but it is most unlikely there were any cognitive ability scores nearly that low. As we note later, they avoided this potential interpretational problem by using mean-centering.
} 
which may or may not be causal effects. In the following we use "partial relationship" to refer to the coefficients $b_{1}$ and $b_{2}$ in the ADD model because they are the "partial coefficients when controlling for the other variable" and we use "conditional partial relationship" (usually shortened to "conditional relationship") to refer to the coefficients $c_{1}$ and $c_{2}$ because they are the "partial coefficients when controlling for the other variable conditioned on that other variable having a specific value."

Psychological researchers have followed several different strategies for talking meaningfully about the results of MMR. We briefly list these strategies and provide a contemporary example or two of each one's appropriate use in the psychological literature.

Two-stage modeling Cohen (1978) recommended a hierarchical test of first ADD, then MMR. The coefficients $b_{1}$ and $b_{2}$ from ADD represent the partial relationships and the coefficient $c_{3}$ from MMR represents the interaction qualifying those two effects (e.g., Kitayama et al., 2015). Some researchers decide the partial relationships are not interesting because they are qualified by an interaction so they only report and discuss $c_{3}$ from MMR (e.g., Duffy \& Chartrand, 2015) and perhaps at spotlighted (Irwin \& McClelland, 2001) or pick-a-point (Hayes, 2013) values of the other variable such as conditional relationship tests for the slopes of different groups (e.g., Chugani, Irwin, \& Redden, 2015; Duffy \& Chartrand, 2015) or floodlight (Bauer \& Curran, 2005; Spiller, Fitzsimons, Lynch, \& McClelland, 2013) to test all possible simple effects (e.g., Disatnik \& Steinhart, 2015; Martin, Ryan, \& Brooks-Gunn, 2010).

Mean-centering A little more than a decade after Cohen's (1978) recommendations, a number of authors (e.g., Aiken \& West, 1991; Jaccard, Turrisi, \& Wan, 1990; Judd \& McClelland, 1989) suggested mean-centering predictors before multiplying them (e.g., Kenrick et al., 2016; note especially that Riglin et al., 2016, used mean-centering to avoid the interpretational problems inherent in testing the conditional relationship of stress on depression when cognitive ability was zero (i.e., more than seven standard deviations below the mean). The interaction coefficient $c_{3}$ and its tests are invariant to all changes of origin such as mean-centering, but now $c_{1}$ and $c_{2}$ represent the conditional slope of the respective predictor at the mean level of the other predictor. These estimates of the conditional coefficients using mean-centered predictors are often close to but not the same as the partial coefficients $b_{1}$ and $b_{2}$ except when the predictor distributions are exactly symmetric. Note the purpose of the mean-centering recommendation was not to alter multicollinearity but rather to produce meaningful interpretations within the range of the data for $c_{1}$ and $c_{2}$. As Hayes (2013, p. 289) states in his excellent discussion of mean-centering:
I have debunked the myth that mean centering of $\mathrm{X}$ and $\mathrm{M}$ is necessary prior to the estimation of a model with an interaction between X and M. I cannot take credit for this, however, as this myth and it corollaries have been repeatedly debunked in the methodology literature yet doggedly persist in spite of that literature (see e.g., Cronbach, 1987; Echambadi \& Hess, 2007; Edwards, 2009; Friedrich, 1982; Hayes et al., 2012; Irwin \& McClelland, 2001; Kam \& Franzese, 2007; Kromrey \& Foster-Johnson, 1998; Shieh, 2011; Whisman \& McClelland, 2005). To be sure, there are interpretational advantages associated with mean centering, but the differences in model coefficients and standard errors have nothing to do with reduced multicollinearity that results from mean centering.

Orthogonal transformation Smith and Sasaki (1979) and Friedrich (1982) showed there always exist change-of-origin transformations $X^{\prime}=X-j$ and $Z^{\prime}=Z-k$ such that the predictor coefficients using $X^{\prime}$ and $Z^{\prime}$ in MMR will be exactly the same as the predictor coefficients (i.e., the partial relationships) in the ADD model. That is,

$$
\begin{aligned}
& \mathrm{ADD}: \hat{Y}=b_{0}^{\prime}+b_{1} X^{\prime}+b_{2} Z^{\prime} \\
& \mathrm{MMR}: \hat{Y}=c_{0}^{\prime}+b_{1} X^{\prime}+b_{2} X^{\prime}+c_{3} X^{\prime} Z^{\prime} .
\end{aligned}
$$

This transformation may put researchers more at ease because it leaves the predictor coefficients from the ADD model unchanged. Friedrich (1982, p. 812) defines the orthogonal transformation in terms of components of the variancecovariance matrix of the parameter estimates:

$$
\begin{aligned}
& X^{\prime}=X-\frac{-C V\left(c_{2}, c_{3}\right)}{V\left(c_{3}\right)} \\
& Z^{\prime}=Z^{-}-\frac{-C V\left(c_{1}, c_{3}\right)}{V\left(c_{3}\right)},
\end{aligned}
$$

where $C V$ and $V$ represent the covariance and variance, respectively, of the estimated parameters. Unfortunately, we have not been able to find any uses of this extremely helpful transformation. Perhaps this is because most researchers are not familiar with the variance-covariance matrix of coefficients, even though most regression programs easily produce it. We have derived a new version of this orthogonal transformation (details in the Appendix) that should be much easier for researchers to implement. Obtain the coefficients from ADD and MMR as in Eq. 1 above and then compute new variables using the coefficients from those models.

$$
\begin{aligned}
& X^{\prime}=X-\frac{b_{2}-c_{2}}{c_{3}} \\
& Z^{\prime}=Z-\frac{b_{1}-c_{1}}{c_{3}}
\end{aligned}
$$


Using these transformed variables in an MMR produces the results in Eq. 3 above. This transformation prevents misinterpretations of the MMR coefficients of the type made by ISPB and by some researchers. For an example of how not quite getting the conditional relationships correct due to either mean-centering or dummy coding a dichotomous predictor can cause confusion, see the dispute between Crawford, Jussim, and Pilanski (2014) and Nosek and Linder (2014). More importantly for purposes of this commentary, the transformation in Eq. 5 will help us disentangle the effects of correlations among the predictors on the standard errors of coefficients in MMR.

Lance (1988), echoed by Little, Bovaird, and Widaman (2006) and Geldhof et al. (2013), proposed "residual centering" in which the residual product, after predictions using both components, is entered into a regression analysis with the ADD model. This method produces identical regression coefficients and tests for the conditional relationships and product as does the orthogonal transformation. Ato, Galián, and Fernández-Villar (2015) provide a recent example of the use of residual centering in psychology; however, their multicollinearity motivation for using this method is incorrect because the usefulness of residual centering is in the interpretation of the parameters, not in the reduction of multicollinearity.

\section{Numerical example}

We generated a more complex example than that used in ISPB in order to illustrate alternative strategies for interpreting models with interactions and, in particular, the consequences of the various transformations. $X$ and $Z$ in this dataset are substantially correlated $(r=0.50)$ and have skewed distributions (beta-binomial) over 1- to 7-rating scales. We generated 100 observations $^{3}$ with $Y$ as a function of $X, Z$, and $X Z$, added normally-distributed errors, and rescaled to a seven-point rating scale. We now compare the additive multiple regression model with several alternative representations of the moderated multiple regression model for this dataset. Hayes, Glynn, and Huge (2012, see especially Table 1) and Hayes (2013, see especially Table 9.2) provide similar useful illustrations of using different transformations to examine alternative models.

Additive The first row of Table 1 presents the coefficients, standard errors, and variance inflation factors for the ADD model. These coefficients capture the partial slope for each predictor variable when controlling for the other one. The variance inflation factors, $V I F=1.355$, reflect the substantial

\footnotetext{
$\overline{3}$ The dataset is available for download at http://psych.colorado. edu/ mcclella/interactionExample.csv
}

Table 1 Unstandardized regression coefficients, standard errors (in parentheses), and variance inflation factors (VIF, in brackets) for four regression models of the numerical example dataset

\begin{tabular}{llll}
\hline & $\mathrm{X}$ & $\mathrm{Z}$ & $\mathrm{XZ}$ \\
\hline ADD & 0.473 & 0.039 & \\
& $(0.082)$ & $(0.094)$ & \\
MMR & {$[1.335]$} & {$[1.335]$} & \\
Raw & -0.066 & -0.709 & 0.152 \\
& $(0.218)$ & $(0.297)$ & $(0.057)$ \\
MMR & {$[9.894]$} & {$[14.016]$} & {$[32.680]$} \\
Centered & 0.553 & 0.031 & 0.152 \\
& $(0.085)$ & $(0.092)$ & $(0.057)$ \\
MMR & {$[1.526]$} & {$[1.336]$} & {$[1.176]$} \\
Orthogonal & 0.473 & 0.039 & 0.152 \\
& $(0.080)$ & $(0.091)$ & $(0.057)$ \\
& {$[1.335]$} & {$[1.335]$} & {$[1.00]$} \\
\hline
\end{tabular}

$A D D$ additive regression model, $M M R$ moderated multiple regression

correlation $(r=0.50)$ between the two predictor variables. For the ADD model, $R^{2}=0.328$.

MMR — raw The second row of Table 1 presents the same values for the MMR using the original, untransformed or raw values. Adding the product term to the model increases $R^{2}$ significantly $\left(F_{1,96}=7.10, p=0.009\right)$ to 0.374 (so the squared semi-partial correlation equals $s r^{2}=0.374-0.328=0.046$ ). Note that the coefficients for $X$ and $Z$ are very different from those in the additive model and the variance inflation factors are enormous. Such high VIFs in an additive model would generally indicate extreme trouble with accuracy of estimation. ISPB cite Marquardt's (1970) recommendation that VIF should not exceed 10. However, this recommendation is a completely inappropriate generalization of a heuristic for an additive model to a moderated model (Irwin \& McClelland, 2001) because it confuses structural with stochastic relationships. We shall see these coefficients are estimated accurately and consistently across all models, despite the high values of VIF.

We know from Eq. 2 that the slope relating $X$ to $Y$ as a function of $Z$ is defined by $\left(c_{1}+c_{3} Z\right)$. We can graph this linear relationship with $Z$ as a straight line along with its $95 \%$ confidence bands, as in Fig. 1. Spiller et al. (2013) provide the details of constructing this graph and describe it as a "floodlight" illuminating all possible tests of the simple slope of for $X$ for any fixed level of $Z$ (for a use of this graph in psychology, see Martin, Ryan, \& Brooks-Gunn, 2010, see also examples in Hayes, 2013). The coefficient for $X$ in the raw MMR equals -0.066 and represents the conditional relationship or equivalently the slope relating $X$ to $Y$ when and only when $Z=$ 0 (the left-most mark on the line in Fig. 1). Because $Z$ values were on a rating scale from 1 to 7 , this is a predicted slope for $X$ beyond the range of the data, as indicated by the dotted lines in Fig. 1. Also note that the confidence band is wider at that 


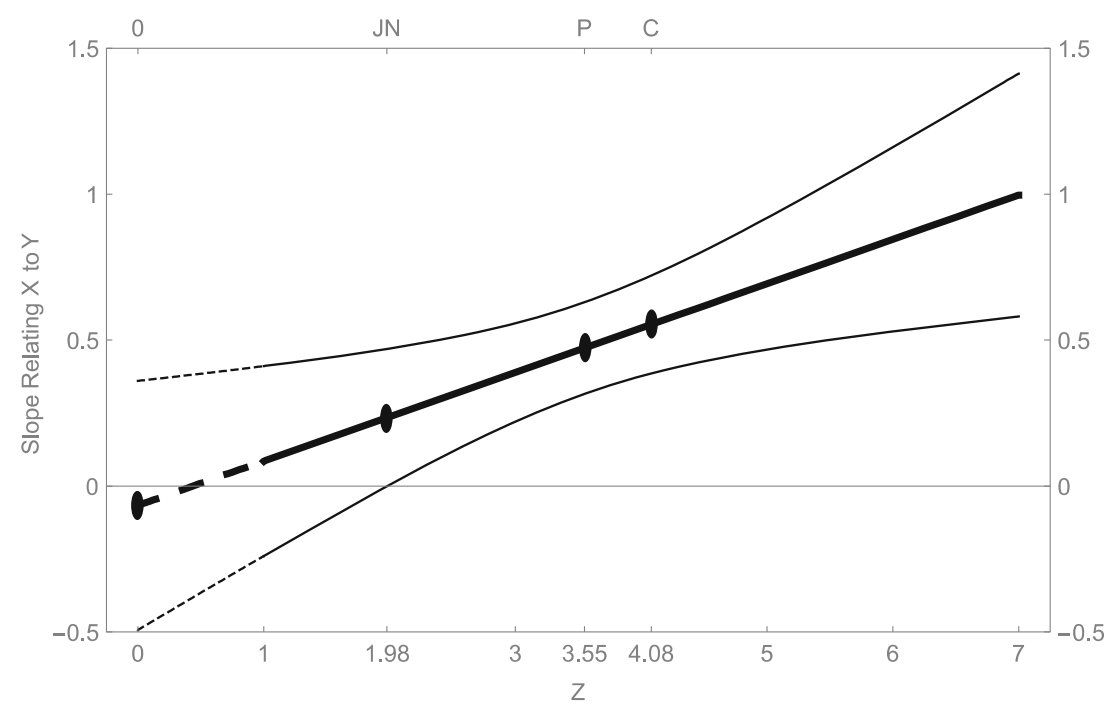

Fig. 1 "Spotlight graph" of the linear relationship between the conditional slopes and the moderator, with its $95 \%$ confidence band. Highlighted points are described in the text

point than at any other value of $Z$ in the graph, again because it is a prediction beyond the range of the data, far from the center of the data values. The changed value of the coefficient for $X$ and its large standard error have nothing to do with multicollinearity (i.e., the high VIFs nominally computed) and everything to do with the meaning of the coefficient for $X$ changing and being outside the range of the data. A similar analysis shows that the coefficient for $Z$ is also meaningless and its standard error is large because it is outside the range of the data and far from the center of the data values.

MMR - mean-centered The analysis in the third row of Table 1 uses mean-centered predictors in the moderated multiple regression model. That is, the predictors are

$X^{\prime}=X-\bar{X}=X-4.87$

$Z^{\prime}=Z-\bar{Z}=Z-4.08$.

Now the coefficient for $X^{\prime}$ equals 0.553 and represents the conditional relationship between $X$ and $Y$ when and only when $Z$ equals its mean 4.08 or, equivalently, when $Z^{\prime}=0$. The rightmost mark, indicated by " $C$ " on the upper axis of Fig. 1, represents this coefficient. Its standard error is noticeably smaller than that for the estimate in the raw MMR model simply because the parameter estimate is for a conditional relationship more in the middle of the data. The variance inflation factors are now at levels that should not frighten anyone, even though they are irrelevant for the statistical test of the interaction. Note, as proved many times before, the coefficient for the product term and its standard error are identical for both the raw and mean-centered MMR models (Cohen, 1978), despite the large differences in the VIFs for the two models.
MMR - orthogonal transformation The fourth row of Table 1 reports the results for orthogonally transformed variables, using the parameter values for ADD and the raw MMR in the first two rows:

$$
\begin{aligned}
& X^{\prime}=X-\frac{b_{2}-c_{2}}{c_{3}}=X-\frac{0.039-(-0.709)}{0.152}=X-4.92 \\
& Z^{\prime}=Z-\frac{b_{1}-c_{1}}{c_{3}}=Z-\frac{0.473-(-0.066)}{0.152}=Z-3.55
\end{aligned}
$$

Note that the coefficients for the predictors $X$ and $Z$ are now identical to those coefficients in the ADD model. Indeed, they represent the same partial relationships between the predictors and $Y$. Also note that their standard errors are ever so slightly smaller in the orthogonal MMR model than in the ADD. This will be the case whenever and to the extent that the test of the interaction has $|t|>1.0$ (i.e., the benefit of the reduction in the mean sum of squares is greater than the loss of one degree of freedom). Furthermore, as shown by Friedrich (1982), the standard errors are the smallest possible standard errors for those coefficients. Figure 1 illustrates this minimum by showing the narrowness of the confidence bands for the mark at $Z=$ 3.55 , under the " $P$ " on the upper axis. Hence, a substantial interaction effect, despite the structural relationship between the predictor variables and their product, actually improves the accuracy of the estimates of the partial relationships. Of course, the estimate, standard error, and test of the interaction coefficient remain unchanged. Also, the $R^{2}=0.374$ for the overall model remains the same as it was for the Raw and Mean-Centered analyses. We emphasize that if one only wants to report and discuss the interaction, then this transformation is not needed. However, if there is any interest in reporting and discussing the same partial relationships as in the ADD model, this orthogonal transformation avoids all confusion in understanding MMR results. Finally, note that the VIF for the 
product term now equals 1.0, indicating that it is completely independent of or orthogonal to the estimates of the partial relationships. The VIFs for the two predictor variables are now identical to their values in the ADD model. That is, the only remaining correlation in the model is attributable to the initial correlation between the two predictors.

Note that in the three moderated models of Table 1, the $V I F$ s for the product term are $32.680,1.176$, and 1.00; however, the regression coefficient for the product and its standard error are identical for all three models. This demonstrates that multicollinearity is completely irrelevant for the accurate estimation and testing of the interaction. Rules-of-thumb learned about the effects of stochastic relationships among variables in additive regression models do not generalize to the structural relationships in moderated multiple regression models (Irwin \& McClelland, 2001).

As a final note on Fig. 1, observe that the $95 \%$ confidence bands intersect the line for a slope of 0 at $Z=1.92$. To the left of that point, no conditional slopes for $X$ would be statistically significant at the conventional criterion of $p<0.05$, and to the right of that point all conditional slopes would be significant. Johnson and Neyman (1936) first derived that point (or points, there may be an upper limit in some cases) as the simple slope that is just significant (i.e., $p=0.05$ ). See Bauer and Curran (2005), Hayes (2013), Hayes and Matthes (2009), and Spiller et al. (2013) for details of computing the Johnson-Neyman point(s). It is identified in Fig. 1 by " $J N$ " on the upper axis. Recent examples in psychology identifying the JohnsonNeyman point(s) include Beach et al. (2012) and Bushman et al. (2012).

Figure 1 makes clear that all the different MMR analyses in Table 1 simply spotlight different slopes for $X$ conditional on different levels of $Z$. The raw MMR spotlights the simple slope for $X$ when $Z=0$, a value outside the range of the data. The mean-centered MMR analysis spotlights the simple slope when $Z$ equals its mean. The MMR with the orthogonal transform spotlights the original partial relationships. Finally, the Johnson-Neyman point spotlights the value of $Z$ for which the conditional relationship between $X$ and $Y$ is just significant. None of these is more correct than the others, they simply highlight different parts of the elephant that is the interaction.

\section{Extensions}

The example above used continuous variables. Nevertheless, the issues apply whether $X$ and/or $Z$ are dichotomous so long as these variables are numerically coded (e.g., contrast, effect, or dummy codes) in a regression analysis. Especially when the data are not from a balanced, orthogonal design, all of the transformations above are useful. For example, when $\mathrm{Z}$ represents two groups, a spotlight analysis first using the dummy codes $(0,1)$ and a second analysis using $(1,0)$ provides tests of the conditional slopes within each group (e.g., Duffy \& Chartrand, 2015)

Another interesting extension is to quadratic regression in which $X$ effectively moderates itself. Consider the linear and quadratic models:

$$
\begin{aligned}
& \text { L I N : } \quad \hat{Y}=d_{0}+d_{1} X \\
& \text { QUAD : } \hat{Y}=e_{0}+e_{1} X+e_{2} X^{2} .
\end{aligned}
$$

The coefficient $d_{1}$ is the simple linear relationship of $X$ and $Y$. The coefficient $e_{2}$ indicates the degree to which that linear relationship depends on the level of $X$. Using the raw variable, $e_{1}$ estimates the conditional relationship when and only when $X=0$, which may or may not be within the range of the data or relevant. All the tools above-mean-centering, orthogonal transformation, spotlight or pick-a-point, floodlight, and Johnson-Neyman - may be used to better understand the nature of the quadratic relationship. Miller, Stromeyer, and Schwieterman (2013) provide details of the extension of some of the tools described here for interpreting quadratic models; Foster, Shiverdecker, and Turner (2016) use these tools to interpret quadratic models for psychological variables. For the quadratic model, the conditional slope is slightly more complicated than it was for MMR so the orthogonal transformation is slightly different. The slope is the first derivative, which equals $e_{1}+2 e_{2} X$. That is, the slope relating $X$ to $Y$ varies as a function of $X$. The transformation

$X^{\prime}=X^{-} \frac{\left(d_{1}-e_{1}\right)}{2 e_{2}}$

yields the following quadratic model in which the coefficient for $X^{\prime}$ will be identical to its value in the simple linear model:

$\hat{Y}=e_{0}^{\prime}+d_{1} X^{\prime}+e_{2} X^{\prime 2}$.

\section{Correlation between $X$ and $Z$}

The results for the orthogonal transformation show that the only correlation of importance in MMR is that between the predictor variables. The consequences of this correlation for estimating and interpreting the partial regression coefficients are exactly as they are for additive multiple regression. Correlated predictors inflate the standard errors for the coefficients $b_{1}$ and $b_{2}$ in the ADD model, and, more importantly, require that the partial relationships be interpreted as the relationship of one predictor with the dependent variable when the other predictor is partialed out, controlled, or held constant. These consequences for the partial relationships are identical whether the product term is added to the model or not. 
Of greater interest in MMR is the possible effect of correlation between the predictors on the statistical power for detecting the interaction. Formally, all else being equal, the standard error for $c_{3}$ will be smaller and the statistical power greater when the residual variance of the product is larger. McClelland and Judd (1993) in their mathematical analysis of the statistical power problems inherent in MMR with continuous predictors provide a formula for the residual variance of the product. When the predictors are jointly symmetric, the variance of the product reduces to

$$
\begin{aligned}
V\left(X^{\prime}, Z^{\prime}\right)= & V\left(X^{\prime}\right) V\left(Z^{\prime}\right) \\
& +C V\left(X^{\prime 2}, Z^{\prime 2}\right)-C V^{2}\left(X^{\prime}, Z^{\prime}\right)
\end{aligned}
$$

Usually, as the correlation between two variables increases, the covariance of their squares increases more rapidly than the square of their covariance. Hence, increasing correlation will usually increase the variance of the product, which in turn reduces the standard error and increases the statistical power for the interaction. Joint symmetry is a strong condition. McClelland and Judd (1993) provide the more general equation not assuming joint symmetry, but analysis of the effects of increased predictor correlation in the more general case does not seem tractable nor are those effects likely to be large. We simply make the point that unlike in additive regression, correlation among the predictors by creating cases extreme on both $X$ and $Y$ can sometimes improve the power of moderated regression for detecting an interaction.

\section{Standardized regression coefficients in MMR}

Standardized regression coefficients, often called "betaweights," are meant to represent the regression coefficients one would obtain if all the variables were first converted to $z$-scores. The first step in computing a $z$-score is to meancenter the variable and the second step is to divide by the variable's standard deviation. In other words, $z$-scores are always mean-centered! It is therefore very confusing that ISPB purport to compare the standardized regression coefficients for raw and mean-centered predictors. If the analysis is done correctly, the same standardized regression coefficients should be obtained whether one starts with raw or mean-centered predictors. However, the $\beta$ 's reported in their Table 3 are decidedly not equal to one another for the raw and meancentered analyses. The problem is that most regression programs use a shortcut for computing standardized regression coefficients that assumes an additive model. The shortcut is inappropriate because it ignores the fact that the product of two $z$-scores does not in general equal the $z$-score of the product. This problem has been noted by Aiken and West (1991), Friedrich (1982), Irwin and McClelland (2001) —all cited by ISPB - and many others (e.g., Hayes, 2013). Hayes (2013) is unambiguous on this matter: "standardized regression coefficients generated by your OLS regression program should never be reported, and you should never probe an interaction using these regression coefficients [emphasis in original, $\mathrm{p}$. 295]." These sources generally recommend against the use of standardized regression coefficients in MMR because they do not have the meaning most researchers believe they have. Footnote 2 in ISBP suggests a reviewer noted this problem with standardized scores in MMR. As that reviewer suggested, researchers using moderated multiple regression should use a measure such as the squared partial correlation $p r^{2}$ or the squared semi-partial correlation $s r^{2}$ to compare effect sizes of the variables instead of standardized regression coefficients.

If one absolutely must have standardized regression weights, the solution, as first detailed by Friedrich (1982), is to standardize the predictors, compute the product from the standardize predictors, and perform a multiple regression on those variables; then the reported regression weights will correspond to standardized regression coefficients. Allen et al. (2015), Keller et al. (2005), and Sherman et al. (2015) are successful examples of this approach in psychology. In contrast, ISPB used the incorrect method for computing standardized regression weights. For both analyses reported in Table 3 of ISPB, when computed according to the correct method, the standardized regression coefficients for the two predictors and the interaction are, respectively, $0.422,0.554$, and -0.082 . For the original variables none of the reported $\beta$-weights are correct; for the centered variables, the interaction $\beta$-weight is incorrect. Because ISPB used the same incorrect method for computing the standardized regression coefficients in their simulations, the results in their Figures 2, 3, and 4 are incorrect and should be completely ignored.

\section{Summary and recommendations}

Multicollinearity, whether it be ISPB's "micro" or "macro" varieties, is a red herring in the hunt for interactions in moderated multiple regression. Researchers using MMR need not compute any multicollinearity diagnostics nor worry about it at all. They need not use mean-centering or the orthogonal transformation or do anything else to avoid the purported problems of multicollinearity. The only purpose of those transformations is to facilitate understanding of MMR models.

We offer simple recommendations for analyzing and reporting moderated multiple regression models. The motivation for these recommendations is to enhance the interpretation of MMR and has nothing to do with modifying supposed multicollinearity.

1. Using original variables fit the model $\hat{Y}=c_{0}+c_{1} X+c_{2} Z+$ $c_{3} X Z$. 
2. If the only interest is the interaction, then report $c_{3}$, its test, and an effect size measure; and report $R^{2}$ and its test for the overall model.

3. If there is interest in reporting and testing any spotlighted conditional relationships such as at the mean of the other variable, or a value of the other variable of particular practical or theoretical interest, or the original partial relationships, then use one or more of the transformations discussed above. In general, to estimate and test the conditional relationship of $X$ (i.e., the slope relating $X$ to $Y$ ) when $Z=k$, then use $Z^{\prime}=Z-k$ to fit the model $\hat{Y}=c_{0}+$ $c_{1} X+c_{2} Z^{\prime}+c_{3} X Z^{\prime}$, in which $c_{1}$ represents the desired conditional relationship. Useful values of $k$ include the mean of $Z$ as well as any specific values of theoretical or practical importance. Hayes (2013, p. 239), Irwin and McClelland (2001), and Spiller et al. (2013) recommend against the common practice of setting $k$ at one standard deviation above or below the mean because it impedes generalization across samples. Instead they recommend testing specific conditional relationships that are meaningful in terms of the original metric.

4. Finally, researchers who wish to examine all possible conditional relationships or to help their readers who might want to consider other conditional relationships, should construct the floodlight graph (e.g., Fig. 1), according to the directions of Bauer and Curran (2005) or Spiller et al. (2013), and identify the Johnson-Neyman point(s) defining regions of statistical significance.

Following these recommended steps yields MMR models that are easy to report and to understand. Additionally, these models will be easier to generalize across studies, leading to a cumulative understanding of the processes underlying the moderation.

Acknowledgments We would like to thank the people who helped us develop these ideas in discussions and who made suggestions for improvement in the presentation: Charles Judd, Andrew Hayes, John Lynch, Jr., Philip Fernbach, Kris Preacher, and Stephen Spiller. The focus of Disatnik and Sivan (2016) is also the unimportance of multicollinearity concerns in moderated multiple regression analysis, but the presentation and examples here do not duplicate that paper.

\section{Appendix}

From Friedrich (1982) we know that there exist variables $X^{\prime}=X-j$ and $Z^{\prime}=Z-k$ such that both variables are independent of their product $X^{\prime} Z^{\prime}$. Given that we know that changes of origin do not affect the partial regression coefficients in additive regression (only the intercept changes), we know the additive model will be:

$\hat{Y}=b_{0}^{\prime}+b_{1} X^{\prime}+b_{2} Z^{\prime}$ where $b_{1}$ and $b_{2}$ are identical to the coefficients obtained from the additive regression model using the original, untransformed variables. Given that the product is independent of both $X^{\prime}$ and $Z^{\prime}$, adding the product to the model will not change the partial regression coefficients, so that in the MMR model

$\hat{Y}=c_{0}^{\prime}+c_{1}^{\prime} X^{\prime}+c_{2}^{\prime} Z^{\prime}+c_{3} X^{\prime} Z^{\prime}$

the interaction coefficient is unchanged and the predictor variable coefficients must be the same as in the Eq. A.1. Hence, $c_{1}^{\prime}=b_{1}$ and $c_{2}^{\prime}=b_{2}$. The equation for the simple slope when $Z=k$ is

$c_{1}^{\prime}=c_{1}+c_{3} k$,

and after substituting for $c_{1}^{\prime}$ becomes

$b_{1}=c_{1}+c_{3} k$.

Finally, to find $k$ we only need to solve the above equation for $k$ :

$k=\frac{b_{1}-c_{1}}{c_{3}}$.

Similarly,

$j=\frac{b_{2}-c_{2}}{c_{3}}$.

All the coefficients necessary for computing the transformation are available from the original ADD and MMR regressions using the untransformed variables.

\section{References}

Aiken, L. S., \& West, S. G. (1991). Multiple regression: Testing and interpreting interactions. Newbury Park, CA: Sage.

Allen, B., Jennings, J. R., Gianaros, P. J., Thayer, \& Manuck, S. B. (2015). Resting high-frequency heart rate variability is related to resting brain perfusion. Psychophysiology, 52, 277-287.

Ato, E., Galián, M. D., \& Fernández-Villar, M. A. (2015). The moderating role of children's effortful control in the relation between marital adjustment and parenting. Journal of Child and Family Studies, 24, 3341-3349.

Bauer, D. J., \& Curran, P. J. (2005). Probing interactions in fixed and multilevel regression: Inferential and graphical techniques. Multivariate Behavioral Research, 40, 373-400.

Beach, S. R. H., Lei, M. K., Brody, G. H., Simons, R. L., Cutrona, C., \& Philibert, R. A. (2012). Genetic moderation of contextual effets on negative arousal and parenting in African-American parents. Journal of Family Psychology, 26, 45-55.

Bushman, B. J., Giancolo, P. R., Parrott, D. J., \& Roth, R. M. (2012). Failure to consider future consequences increases the effects of alcohol on aggression. Journal of Experimental Social Psychology, 48, 591-595.

Chugani, S. K., Irwin, J. R., \& Redden, J. P. (2015). Happily ever after: The effect of identity-consistency on product satiation. Journal of 
Consumer Research. doi:10.1093/jcr/ucv040. Advanced online access.

Cohen, J. (1978). Partial products are interactions; partialed powers are curve components. Psychological Bulletin, 85, 858-866.

Crawford, J. T., Jussim, L., \& Pilanski, J. M. (2014). How (not) to interpret and report main effects and interactions in multiple regression: Why Crawford and Planski did not actually replicate Lindner and Nosek (2009). Political Psychology, 35, 857-862.

Cronbach, L. J. (1987). Statistical tests for moderator variables: Flaws in analyses recently proposed. Psychological Bulletin, 102(3):414417.

Disatnik, D., \& Sivan, L. (2016). The multicollinearity illusion in moderated regression analysis. Marketing Letters, 27, 403-408.

Disatnik, D., \& Steinhart, Y. (2015). Need for cognitive closure, risk aversion, uncertainty changes, and their effects on investment decisions. Journal of Marketing Research, 52, 349-359.

Duffy, K. A., \& Chartrand, T. L. (2015). The extravert advantage: How and when extraverts build rapport with other people. Psychological Science, 26, 1795-1802.

Echambadi, R., Hess, J. D. (2007). Mean-centering does not alleviate collinearity problems in moderated multiple regression models. Marketing Science, 26(3):438-445.

Edwards, J. R. (2009). Seven deadly myths of testing moderation in organizational research. In C. E. Lance \& R. J. Vanderberg (Eds.), Statistical and methodological myths and urban legends (pp. 143164). New York, NY: Routledge.

Foster, J. D., Shiverdecker, \& Turner, I. N. (2016, online). What does the narcissistic personality inventory measure across the total score continuum? Current Psychology (online at http://link.springer. com/article/10.1007/s12144-016-9407-5/fulltext.html).

Friedrich, R. J. (1982). In defense of multiplicative terms in multiple regression equations. American Journal of Political Science, 26, 797-833.

Geldhof, G. J., Pornprasertmanit, S., Shoemann, A. M., \& Little, T. D. (2013). Orthogonalizing through residual centering: Extended applications and caveats. Educational and Psychological Measurement, 73, 27-46.

Hayes, A. F. (2013). Introduction to mediation, moderation, and conditional process analysis: A regression-based approach. New York: The Guildford Press.

Hayes, A. F., Glynn, C. J., \& Huge, M. E. (2012). Cautions regarding the interpretations of regression coefficients and hypothesis tests in linear models with interacdtions. Communication Methods and Measures, 6, 1-11.

Hayes, A. F., \& Matthes, J. (2009). Computational procedures for probing interactions in OLS and logistic regression: SPSS and SAS implementations. Behavior Research Methods, 41, 924-936.

Iacobucci, D., Schneider, M. J., Popovich, D. L., \& Bakamitsos, G. A. (2016). Mean centering helps alleviate "micro" but not "macro" multicollinearity. Behavioral Research Methods. (this issue).

Irwin, J. R., \& McClelland, G. H. (2001). Misleading heuristics and moderated multiple regression models. Journal of Marketing, 38, $100-109$.

Jaccard, J., Turrisi, R., \& Wan, C. K. (1990). Interaction effects in multiple regression. Newbury Park, CA: Sage.

Johnson, P. O., \& Neyman, J. (1936). Tests of certain linear hypotheses and their application to some educational problems. Statistical Research Memoirs, 1, 57-93.

Judd, C. M., \& McClelland, G. H. (1989). Data analysis: A modelcomparison approach. San Diego, CA: Harcourt Brace Jovanovich.

Kam, C. D., \& Franzese, R. J. (2007). Modeling and interpreting interactive hypotheses in regression analysis. Ann Arbor, MI: University of Michigan.

Keller, M. C., Fredrickson, O. Y., Ybarra, O., Côté, S., Johnson, K., Mikels, J., ... Wager, T. (2005). A warm heart and a clear head:
The cognition effects of weather on mood and cognition. Psychological Science, 16, 724-731.

Kenrick, A. C., Sinclair, S., Richeson, J., Verosky, S. C., \& Lyn, J. (2016). Moving while black: Intergroup attitudes influence judgments of speed. Journal of Experimental Pscyhology: General, 145, 147154.

Kitayama, S., Park, J., Boylan, J. M., Miyamoto, Y., Levine, C. S., Markus, H. R., ... Ryff, C. D. (2015). Expression of anger and ill health in two cultures: An examination of inflammation and cardiovascular risk. Psychological Science, 26, 211-220.

Kromrey, J. D., Foster-Johnson, L. (1998). Mean centering in moderated multiple regression: Much ado about nothing. Educational and Psychological Measurement, 58(1):42-67.

Lance, C. E. (1988). Residual centering, exploratory moderator analysis, and decomposition of effects in path models containing interactions. Applied Psychological Measurement, 12, 163-175.

Little, T. D., Bovaird, J. A., \& Widaman, K. F. (2006). On the merits of orthogonalizing powered and product terms: Implications for modeling interactions among latent variables. Structural Equation Modeling, 13, 497-519.

Marquardt, D. W. (1970). Generalized inverses, ridge regression, biased linear estimation, and nonlinear estimation. Technometrics, 12, 591612.

Martin, A., Ryan, R., \& Brooks-Gunn, J. (2010). When father's supportiveness matters most: Maternal and paternal parenting and children's school readiness. Journal of Family Psychology, 24, 145-155.

McClelland, G. H., \& Judd, C. J. (1993). Statistical difficulties of detecting interactions and moderator effects. Psychological Bulletin, 114, 376-390.

Miller, J. W., Stromeyer, W. R., \& Schwieterman, M. A. (2013). Extensions of the Johnson-Neyman Technique to linear models with curvilinear effects: Derivations and analytical tools. Multivariate Behavioral Research, 48, 267-300.

Nosek, B. A., \& Linder, N. M. (2014). Replication actually: Comment on Crawford and Pilanski (2014). Political Psychology, 35, 853-855.

Riglin, L., Collishaw, S., Shelton, K. H., McManus, I. C., Ng-Knight, T., Sellers, R., ... Rice, F. (2016). Higher cognitive ability buffers stress-related depressive symptoms in adolescent girls. Development and Psychopathology, 28, 97-109.

Saunders, D. R. (1955). The "moderator variable" as a useful tool in prediction. In Proceedings of the 1954 invitational conference on testing problems (pp. 54-58). Princeton, NJ: Educational Testing Service.

Saunders, D. R. (1956). Moderator variables in prediction. Educational and Psychological Measurement, 16, 209-222.

Sherman, G. D., Lerner, J. S., Josephs, R. A., Renshon, J., \& Gross, J. J. (2015). The interaction of testosterone and cortisol is associated with attained status in male executives. Journal of Personality and Social Psychology. doi:10.1037/pspp0000063. Advanced online publication.

Shieh, G. (2011). Clarifying the role of mean centring in multicollinearity of interaction effects. British Journal of Mathematical and Statistical Psychology, 64(3):462-477.

Smith, K. W., \& Sasaki, M. S. (1979). Decreasing multicollinearity: A method for models with multiplicative functions. Sociological Methods \& Research, 8, 35-56.

Spiller, S. A., Fitzsimons, G. J., Lynch, J. G., \& McClelland, G. H. (2013). Spotlights, floodlights, and the magic number zero: Simple effects tests in moderated regression. Journal of Marketing Research, 50, 277-288.

Whisman, M. A., McClelland, G. H. (2005). Designing, testing, and interpreting interactions and moderator effects in family research. Journal of Family Psychology, 19(1):111-120 